\title{
Nanoparticle-based drug delivery to improve the efficacy of antiretroviral therapy in the central nervous system
}

This article was published in the following Dove Press journal:

International Journal of Nanomedicine

7 April 2014

Number of times this article has been viewed

\author{
Maria João Gomes' \\ José das Neves ${ }^{1,2}$ \\ Bruno Sarmento ${ }^{1,2}$ \\ 'Instituto de Engenharia Biomédica \\ (INEB), Porto, Portugal; ' Instituto de \\ Investigação e Formação Avançada \\ em Ciências e Tecnologias da Saúde \\ (IINFACTS), Instituto Superior de \\ Ciências da Saúde-Norte, CESPU, \\ Gandra, Portugal
}

\begin{abstract}
Antiretroviral drug therapy plays a cornerstone role in the treatment of human immunodeficiency virus (HIV)/acquired immunodeficiency syndrome patients. Despite obvious advances over the past 3 decades, new approaches toward improved management of infected individuals are still required. Drug distribution to the central nervous system (CNS) is required in order to limit and control viral infection, but the presence of natural barrier structures, in particular the blood-brain barrier, strongly limits the perfusion of anti-HIV compounds into this anatomical site. Nanotechnology-based approaches may help providing solutions for antiretroviral drug delivery to the CNS by potentially prolonging systemic drug circulation, increasing the crossing and reducing the efflux of active compounds at the blood-brain barrier, and providing cell/tissue-targeting and intracellular drug delivery. After an initial overview on the basic features of HIV infection of the CNS and barriers to active compound delivery to this anatomical site, this review focuses on recent strategies based on antiretroviral drug-loaded solid nanoparticles and drug nanosuspensions for the potential management of HIV infection of the CNS.
\end{abstract}

Keywords: HIV/AIDS, blood-brain barrier, protease inhibitors, efflux transporters, drug targeting

\section{Introduction}

There are an estimated 35.3 million people infected with human immunodeficiency virus (HIV) worldwide, according to the latest numbers from the Joint United Nations Programme on HIV/AIDS (UNAIDS). ${ }^{1}$ Although the number of new infections has shown a trend of decrease since 2001, the number of infected individuals keeps on growing, mainly because of the success of and increasing access to antiretroviral drugs. The introduction of combination antiretroviral therapy (cART) around 1996 had a huge impact on the management of acquired immunodeficiency syndrome (AIDS), and resulted in increased quality of life and lifespan of seropositive individuals. ${ }^{2,3}$ However, several limitations of currently available drugs, namely their toxicity and poor pharmacokinetics, the need to be used for prolonged periods of time (if not chronically), and the emergence of viral resistance, still jeopardize the optimal efficacy of cART. ${ }^{4}$ Of particular importance is the poor bioavailability of several anti-HIV drugs at viral reservoir sites, such as the central nervous system (CNS), in special brain macrophages and microglia cells, when using available dosage forms. Low cerebrospinal fluid:blood plasma (CSF:BP) concentration-ratio values have been reported for various drugs commonly used in the management of HIV/AIDS. ${ }^{5}$ For example, protease inhibitors (PIs) bind extensively to plasma proteins, and are substrates of permeability glycoprotein (P-gp) and other important efflux transporters present at the blood-brain barrier (BBB),
Correspondence: Bruno Sarmento Instituto de Engenharia Biomédica (INEB), Rua do Campo Alegre, 823 ,

4I50-180 Porto, Portugal

Tel +35I 226074900

Fax +35I 226094567

Email bruno.sarmento@ineb.up.pt
International Journal of Nanomedicine 2014:9 1757-1769 1757

Dovepress (c) (7) 2014 Gomes et al. This work is published by Dove Medical Press Limited, and licensed under Creative Commons Attribution - Non Commercial (unported, v3.0) (c) (i) \&. 2014 Gomes et al. This work is published by Dove Medical Press Limited, and licensed under Creative Commons Attribution - Non Commercial (unported, v3.0) permission from Dove Medical Press Limited, provided the work is properly attributed. Permissions beyond the scope of the License are administered by Dove Medical Press Limited. Information on how to request permission may be found at: http://www.dovepress.com/permissions.php 
which combined severely limit their uptake into the brain. ${ }^{6}$ Apart from impairing the elimination of the virus from the CNS, the low penetration ability of the BBB by antiretroviral drugs is associated with higher CSF viral loads, which can be critical in the development of neurological disorders. ${ }^{7}$

The use of drug nanocarriers has been advocated as potentially beneficial in delivering antiretroviral drugs across the BBB and into the CNS. ${ }^{8-13}$ Nanotechnology-based systems may additionally provide interesting features, such as enhanced intestinal absorption (following oral administration), improved toxicity profiles, increased drug stability, prolonged drug residence in the body (namely at the CNS), circumvention of efflux pumps (including those at the BBB), and selective drug delivery to specific cells (eg, HIV-target cells). Even if other types of nanocarriers have also been proposed for the management of HIV infection of the CNS, such as dendrimers, ${ }^{14}$ nanoemulsions, ${ }^{15,16}$ liposomes, ${ }^{17}$ micelles, ${ }^{18}$ and nanogels, ${ }^{19}$ this review will focus on solid nanoparticles (NPs) of polymeric, macromolecular, lipid, or metallic nature. Also, strategies based on antiretroviral drug nanosuspensions are overviewed.

\section{HIV and the CNS: infection and barriers to viral eradication}

Lentiviruses, such as HIV, are able to infect all structures in the nervous system. Soon after primary viral transmission, $\mathrm{HIV}$ is able to invade the CNS by means of peripherally infected leukocytes, mainly monocytes. These cells are able to cross the BBB, acting as "Trojan horses". Other mechanisms, such as direct infection or transcytosis of endothelial cells, are also possible, but usually considered of minor importance. ${ }^{20}$ Once at the CNS, the virus is able to replicate and further infect macrophages, astrocytes, $\mathrm{CD}^{+} \mathrm{T}$ cells, and microglia, thus triggering the release of a cascade of host-derived inflammatory molecules and HIV-encoded neurotoxic proteins (eg, gp-120, transactivator of transcription [Tat], and Vpr). ${ }^{21}$ In particular, macrophages and microglia seem to be the most important cell populations sustaining HIV infection of the CNS. ${ }^{20}$ Inflammation and cytotoxicity often lead to neurocognitive and motor disorders, even in patients undergoing cART. ${ }^{22}$ Neurocognitive impairment is frequently symptomatic (mostly memory, learning, and executive function impairment) and associated with initial severe immunosuppression, but usually mild if treatment has been introduced early. ${ }^{23}$ Severe HIV-associated dementia is rare, even though the life expectancy of AIDS patients is increasing, and linked to high virus levels in the $\mathrm{CSF}^{24}$ Viral encephalopathy typically encompasses immune activation (encephalitis), with such histological features as gliosis, microglial nodules, perivascular macrophage accumulation, and the presence of multinucleated giant cells. ${ }^{25}$ Also, inflammation results in increased leakiness of the BBB, which enhances the ability of further viral invasion of the CNS. Adding to the virus effects on the CNS, concomitant use of medication (including some antiretroviral drugs), the presence of other neurological (eg, cerebrovascular disease) and nonneurological (eg, hepatitis $\mathrm{C}$ infection, atherosclerosis) medical conditions, and aging-associated neurodegenerative disease may exacerbate neurological disorders. ${ }^{25}$

The CNS is recognized as an anatomical viral reservoir, where HIV persists in long-lived cells, namely microglia, with more stable kinetics. ${ }^{26}$ This results in increased difficulty of viral eradication by cART and the emergence of different and drug-resistant HIV strains. Added to this, the poor ability of several antiretroviral drugs to penetrate the CNS (Table 1) further limits eradication. ${ }^{5}$ Indeed, antiretroviral drugs may not reach the CNS in sufficient levels or may even be excluded (ie, by such efflux transporters as P-gp) from this site at the BBB level. At the same time, the inflammatory response triggered by HIV infection of the CNS induces changes to the $\mathrm{BBB}$, resulting in tight-junction (TJ)

Table I Selected drugs currently used in combination antiretroviral therapy and their ability to reach the central nervous system, as reflected by the cerebrospinal fluid:blood plasma (CSF:BP) concentration ratio in humans (expressed as mean values or mean range values from cited references)

\begin{tabular}{llll}
\hline Drug classes & Drugs & CSF:BP & References \\
\hline Nucleoside reverse- & Zidovudine & 0.5 & 27 \\
transcriptase inhibitors & Didanosine & 0.21 & 28 \\
& Stavudine & $0.16-0.40$ & 29,30 \\
& Lamivudine & $0.06-0.23$ & 30,31 \\
& Abacavir & $0.18-0.36$ & 32 \\
& Emtricitabine & 0.26 & 33 \\
Nucleotide reverse- & Tenofovir & 0.05 & 33,34 \\
transcriptase inhibitors & & & \\
Nonnucleoside reverse- & Nevirapine & 0.63 & 30 \\
transcriptase inhibitors & Efavirenz & $0.003-0.01$ & 35 \\
& Etravirine & 0.01 & 36 \\
Protease inhibitors & Saquinavir & $\leq 0.002$ & 37,38 \\
& Indinavir & 0.11 & 30 \\
& Ritonavir & $0.001-0.005$ & 38 \\
& Nelfinavir & Undetectable & 39 \\
& & in CSF & \\
& Atazanavir & $0.002-0.014$ & 40 \\
Entry inhibitors & Fosamprenavir & 0.012 & 41 \\
& Darunavir & 0.01 & 42 \\
& Enfuvirtide & Undetectable & 43 \\
& & in CSF & \\
& Maraviroc & 0.028 & 44 \\
& Raltegravir & $0.01-0.61$ & 45 \\
& Dolutegravir & 0.5 & 46 \\
\hline
\end{tabular}


modulation and increased leakiness to different molecules and even circulating cells (eg, HIV-infected monocytes) ${ }^{47}$ Alongside the BBB, other anatomical barriers also pose significant hurdles that limit drug penetration of the CNS, namely the blood-CSF barrier at the choroid plexuses, and to a minor extent the CSF-brain barrier. Blood plasma protein binding has also been held responsible for poor bioavailability of several antiretroviral drugs, such as efavirenz $(99.5 \%$ binding) and various PIs. ${ }^{48}$ Different distribution patterns of HIV were observed at different areas of the CNS of patients undergoing cART, with higher levels of viral ribonucleic acid (RNA) being observed in the caudate nucleus, while the lowest concentrations were observed in the frontal cortex and $\mathrm{CSF}^{49}$ This fact may reflect, at least partially, the heterogeneous distribution of antiretroviral drugs in the CNS. Therefore, strategies enhancing antiretroviral drug levels and distribution in the CNS are required.

\section{Drug delivery to the CNS: focus on nanotechnology}

The CNS is a unique and complex environment with restricted anatomical access, mainly due to such brain barriers as the BBB. ${ }^{50}$ The $\mathrm{BBB}$ is an active, dynamic, and complex interface between the blood and the CNS that has neuroprotective functions, regulates the in-and-out brain transport of different molecules and cells (eg, leukocytes), and maintains the homeostasis of the brain microenvironment. ${ }^{51,52}$ This strict access is mainly due to the presence of TJs between endothelial cells of blood capillaries interfacing with the CNS (Figure 1). ${ }^{53}$ The role of the BBB is crucial for neuronal activity and proper functioning of the CNS. Thus, when drug delivery to this anatomical site is required, overcoming the $\mathrm{BBB}$ blocking action is one of the main hurdles in order to improve and develop effective treatments without the need to administer directly into the CNS or use high drug doses with increased risks of adverse side effects. ${ }^{54}$

There are different options for molecules to cross the $\mathrm{BBB}$, which could be strategically used for drug delivery purposes. In the case of the transport of low-molecular-weight molecules, there are two possibilities: 1) diffusion, either passive or facilitated across aqueous channels, and 2) active transport, which is mediated by such carriers as proteins (carrier-mediated transport). As for macromolecules, their transport includes receptor-mediated transcytosis (RMT), nonspecific adsorptive-mediated transcytosis (AMT), and cell-mediated transcytosis. RMT involves the endocytosis of macromolecules specifically bound to a receptor on the endothelial surface of the BBB, their diffusion across the
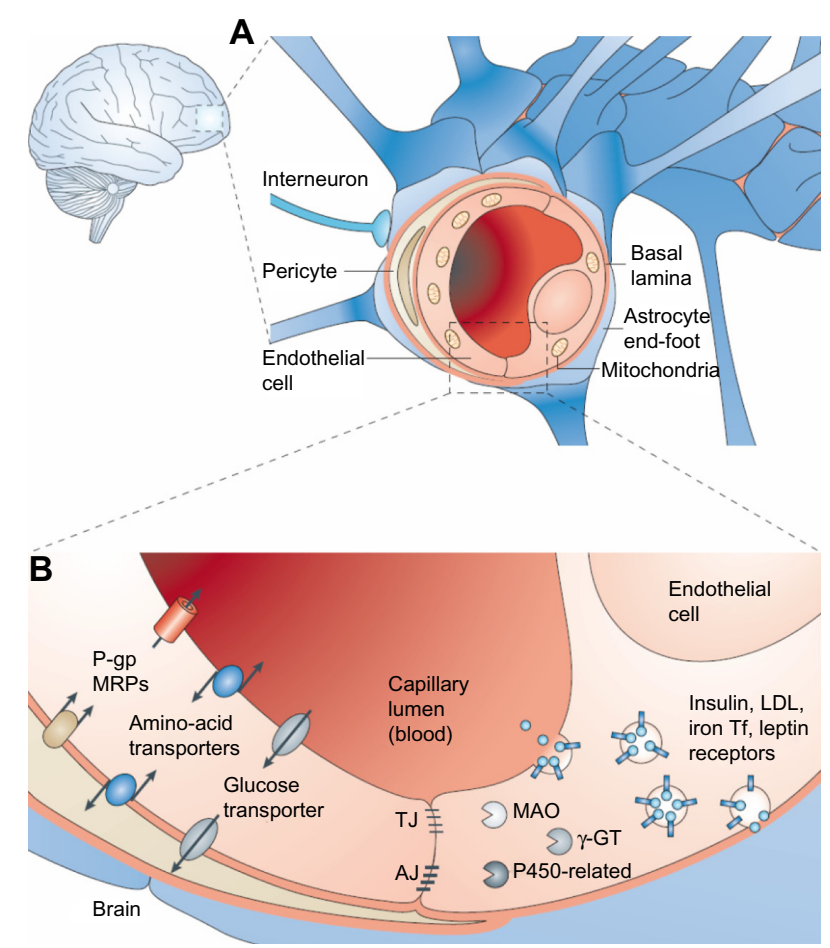

Figure I The blood-brain barrier (BBB).

Notes: $(A)$ The BBB is formed by endothelial cells at the level of the cerebral capillaries. These endothelial cells interact with perivascular elements, such as the basal lamina and closely associated astrocytic end-feet processes, perivascular neurons (represented by an interneuron here), and pericytes to form a functional BBB. (B) Cerebral endothelial cells are unique in that they form complex tight junctions (TJs) produced by the interaction of several transmembrane proteins that effectively seal the paracellular pathway. These complex molecular junctions make the brain practically inaccessible for polar molecules, unless they are transferred by transport pathways of the BBB that regulate the microenvironment of the brain. There are also adherens junctions (AJs), which stabilize cell-cell interactions in the junctional zone. In addition, the presence of intracellular and extracellular enzymes, such as monoamine oxidase (MAO), $\gamma$-glutamyl transpeptidase $(\gamma-\mathrm{GT})$, alkaline phosphatase, peptidases, nucleotidases, and several cytochrome P450 enzymes, endow this dynamic interface with metabolic activity. Large molecules, such as antibodies, lipoproteins, proteins, and peptides can also be transferred to the central compartment by receptor-mediated transcytosis or nonspecific adsorptive-mediated transcytosis. The receptors for insulin, low-density lipoprotein (LDL), iron transferrin (Tf) and leptin are all involved in transcytosis. Reprinted by permission from Macmillan Publishers Ltd: Nat Rev Drug Discov. Cecchelli R, Berezowski V, Lundquist S, et al. Modelling of the blood-brain barrier in drug discovery and development, (C) 2007.53

Abbreviations: P-gp, P-glycoprotein; MRP, multidrug resistance-associated protein family.

endothelium, and exocytosis on the opposite site..$^{50} \mathrm{AMT}$, also known as pinocytosis, is mediated by electrostatic interactions between positively charged ligands and the negatively charged membranes of the BBB. ${ }^{55}$ Cell-mediated transcytosis refers only to immune cell-mediated transport. ${ }^{56}$ Finally, active efflux transport is another important type of molecule transfer across the BBB that limits brain penetration. This last mechanism involves extrusion of drugs from the CNS back to the blood, thus representing a major impediment to drug therapy. ${ }^{52,57}$ Until now, the most extensively characterized efflux transporters at the BBB have been multidrug resistance-associated proteins (MRPs), of which P-gp is the main representative. ${ }^{58-60} \mathrm{P}$-gp has a high affinity for a wide 
range of cationic and lipophilic compounds, and thus limits the transport of many drugs, including cytotoxic anticancer drugs, antibiotics, hormones, and antiretroviral PIs. ${ }^{61,62}$

Among others, nanotechnology-based strategies have been useful in overcoming the BBB, as they can generally improve the permeability characteristics of different pharmacologically active agents, making them able to achieve target sites. ${ }^{63}$ NPs are great examples of drug carriers, due to their versatile and tunable properties, such as large surface:volume ratio, surface charge, and small and controllable size, ${ }^{64}$ which promote their permeation through the $\mathrm{BBB}$ and facilitate drug delivery to the CNS. ${ }^{65}$ Moreover, NPs can be made nontoxic, biodegradable, and biocompatible, depending on proper material selection and manufacturing. ${ }^{52} \mathrm{NPs}$ have also a customizable surface that can be modified in order to target different cells or tissues, according to their final intent. These characteristics can contribute to increased drug bioavailability and decreased peripheral toxicity and side effects. ${ }^{56,57,64,66}$ Charge and hydrophobicity of the NP surface influence the adsorption of plasmatic proteins, and as a consequence their uptake and/or rate of transcytosis. ${ }^{67}$ In the particular case of CNS delivery, NP coating with particular surface stabilizers may be of interest in achieving increased brain drug levels. The best-characterized one is polysorbate 80 , a nonionic surfactant that has been shown to be effective as a brain-delivery enhancer in different types of NPs, such as polymeric NPs ${ }^{68,69}$ and solid-lipid NPs (SLNs).$^{70}$ Once in the blood circulation, polysorbate 80 -coated NPs adsorb different apolipoproteins, thus mimicking lipoproteins in their RMT pathway into the $\mathrm{CNS}{ }^{71}$

Engineered NPs targeting the CNS can be obtained by surface coupling of specific molecules that provide them the ability to overcome the BBB and eventually also reach HIV-reservoir sites, depending on the type of functionalization and incorporated ligands. Targeting mechanisms could either be passive or active. In this last case, transportermediated delivery is a strategy commonly used to allow NPs to cross the BBB based on the principle that peptides or other molecules may use specific transporters expressed on endothelial cells at the BBB. These molecules usually recognize and bind to target receptors or antigens, which may be overexpressed or selectively expressed by particular cells or tissue components. ${ }^{63}$ In the case of the AMT mechanism, the most prominent candidates used as NP surface-targeting moieties are cell-penetrating peptides. These ligands facilitate enhanced intracellular drug delivery through endocytosis or by the formation of a transient structure with the cell membrane. $^{72}$ The HIV-1 Tat peptide is one of the most widely tested cell-penetrating peptides. ${ }^{10}$ This peptide possesses certain regions, known as protein-transduction domains, that can promote migration through biological membranes. The BBB-permeation mechanism is independent of transporters and receptor-mediated endocytosis, but depends on the fusion of $\beta$-galactosidase to the Tat peptide. ${ }^{73,74}$ Another peptide that is also commonly used for achieving brain targeting is glutathione. This endogenous tripeptide possesses antioxidant properties and plays a central role in the detoxification of intracellular metabolites. Glutathione has been shown to possess the ability to enhance drug delivery to the brain as mediated by liposomes. ${ }^{75}$

One of the most widely studied proteins for targeted drug delivery is transferrin (Tf). The transport of this endogenous protein is mediated by the Tf receptor-known to be expressed in the luminal membrane of the capillary endothelium of the $\mathrm{BBB}$, among other body regions - which can also be targeted by specifically designed antibodies. ${ }^{76-78}$ Although the mechanism of this approach is not yet fully understood, results from multiple studies of drug targeting and delivery to the brain with these types of moieties confirm its feasibility (reviewed by De Boer and Gaillard). ${ }^{61}$ As described earlier for the mechanism of polysorbate 80-coated NPmediated CNS drug delivery, apolipoproteins, namely apolipoprotein E (ApoE), are obvious candidates for surface functionalization of NPs. ApoE is involved in the transport of lipids into the brain via low-density lipoprotein receptors, which are essential for maintaining cholesterol homeostasis in the brain. ${ }^{79}$ ApoE not only binds to various receptors on the $\mathrm{BBB}$ (eg, the low-density lipoprotein receptor-related protein [LRP]-1) but also to receptors in other regions of the CNS, which may be an additional advantage for subcompartmental HIV therapy. Both LRP-1 and LRP-2 have been explored in order to target drugs to the brain. ${ }^{80}$

Due to their role in HIV infection of the CNS, monocytes/ macrophages can be interesting targets for therapeutic NPs. Monocytes/macrophages have various surface receptors, namely to mannose residues, which can help them in the process of recognition and endocytosis of NPs. Hence, NPs functionalized with mannose/mannan are better phagocytozed by these cells. ${ }^{81}$ Also, bradykinin type II $\left(\mathrm{B}_{2}\right)$ receptors are primarily expressed in neuronal and vascular tissue, which makes these interesting targets for drug delivery into the CNS. $\mathrm{B}_{2}$-receptor agonists may be effective in targeting the BBB by mediating the opening of TJ (through a calciummediated mechanism) at the brain-microvascular endothelial cell (MEC) interface. In addition, RMP-7, which is a synthetic linear pseudopeptide that functions as a $\mathrm{B}_{2}$-receptor 
agonist, could be used as a targeting BBB molecule. ${ }^{82}$ Furthermore, exposure to an electromagnetic field (EMF) can also augment the permeability of drugs across the BBB, since it could cause significant and temporary alterations in the structure of this natural barrier. Generally, higher EMF power yields increased permeability, but the long-term effects of these changes to the BBB may be hazardous. ${ }^{83}$

As efflux transporters may greatly limit the residence of antiretroviral drugs in the CNS, another possible strategy to enhance inward flux across the BBB is the inhibition of these efflux-transport systems. ${ }^{61}$ As mentioned before, P-gp is the most widely studied representative of all the BBB effluxsystem proteins, ${ }^{84}$ and prevents the accumulation of a remarkably wide range of substrates in the brain endothelial cells through potent adenosine triphosphate-driven pumping. ${ }^{85}$ Therefore, improved drug uptake into the CNS could be achieved by altering the MRP1 function at the BBB, as shown in a pioneer in vivo study by using MRP1-knockout mice, where brain-to-plasma ratios of a considerable number of drugs increased five- to 50-fold. ${ }^{86}$ Consequently, many MRP1 inhibitors have been developed since, ${ }^{87}$ but their toxicity has been an important limitation. ${ }^{88}$ Therefore, other options need to be considered, and small interfering RNA (siRNA) directed against MRP1 has been shown to be promising as a new reversal agent for avoiding the undesired effects of MRP1 inhibitors. ${ }^{89,90}$ In order to improve physical stability, resistance to nuclease degradation, intracellular penetration, and efficacy and toxicity profiles, NPs can be used to deliver siRNA. This is an alternative way to temporarily disable the efflux mechanisms of the BBB and thus reach the CNS. After the administration and silencing effect of siRNA, the required drugs (such as antiretroviral drugs) can be administrated and reach the CNS, thus circumventing MRP1 efflux.

\section{Antiretroviral drug-loaded nanoparticles for CNS delivery}

Various nanocarrier-mediated solutions have been proposed in order to increase antiretroviral drug delivery to the CNS. For example, the ability of nanostructured lipid carriers (NLCs) to potentially mediate the brain delivery of zidovudine, a frequently used nucleoside reverse-transcriptase inhibitor that has related toxic effects and a very short plasma half-life (around 1 hour), was studied in vitro by Joshy and Sharma. ${ }^{91}$ Spherical NLCs comprising a mixture of Compritol ${ }^{\circledR} 888$ ATO (Gattefossé, Saint-Priest, France), stearic acid, and oleic acid and prepared using a solventdiffusion method, with a final size range of 170-500 nm, were developed and shown to be taken up by the $\mathrm{C} 6$ brain cell line after 2 hours of incubation, as assessed by fluorescent microscopy. Rhodamine-loaded NLCs associated with the cells and deposited either on the outer side of the plasmatic membrane or in the cytosol. Although preliminary, the ability of these NLCs to promote intracellular trafficking at a representative cell line of the BBB suggests that these nanocarriers may be a promising system to enhance zidovudine brain uptake. In another study, atazanavir was loaded into stearic acid-based SLNs for enhanced brain delivery by Chattopadhyay et al. ${ }^{92}$ SLNs produced by a thin-film hydration technique based on a microemulsion possessed negative charges (zeta potential of approximately $-18 \mathrm{mV}$ ) and a mean particle-size range between 150 and $250 \mathrm{~nm}$. Besides confirming the low toxicity ppotential of SLNs in hCMEC/ $\mathrm{D}_{3}$ cell line, a human brain microvessel endothelial cell line (HBMEC), up to a concentration corresponding to $200 \mathrm{nM}$ of atazanavir, this group also demonstrated significantly higher drug-permeation ratios through endothelial cell monolayers compared to atazanavir in aqueous solution after 2 hours of incubation. The hCMEC/D 3 cell line, which is an extensively characterized human brain endothelial cell line, mimics most of the key features of the BBB, and thus represents a useful model for screening drug candidates and carrier systems for CNS drug delivery. ${ }^{92,93}$ Despite the interesting in vitro results obtained by this group, future in vivo work is required in order to demonstrate the potential efficacy of proposed SLNs to deliver atazanavir to the brain.

In a study by Kuo and $\mathrm{Su},{ }^{94}$ both polymeric (polybutylcyanoacrylate [PBCA] and methyl methacrylate-sulfopropyl methacrylate [MMA-SPM]) NPs and SLNs (comprising mixtures of tripalmitin and cocoa butter) were developed as carriers for stavudine, delavirdine (a nonnucleoside reversetranscriptase inhibitor), and saquinavir, and characterized for their ability to modulate drug permeability across an in vitro BBB model. Nanocarriers were produced by emulsion polymerization (PBCA NPs), free radical polymerization (MMA-SPM NPs) or microemulsion method (SLNs). The BBB model comprised a dual-chamber setup separated by a confluent HBMEC monolayer on a microporous polycarbonate membrane, and cultured on human fibronectin-coated dishes with endothelial cell medium. These studies showed that even though increases in particle size from 90 to $185 \mathrm{~nm}$ (PBCA NPs), 5 to $70 \mathrm{~nm}$ (MMA-SPM NPs), and 140 to $320 \mathrm{~nm}$ (SLNs) yielded a decrease in the permeability coefficient, all three NP types were efficacious carriers of all tested drugs and improved the BBB permeability by three- to 16-fold compared to the compounds in solution. Later, Kuo and Lee ${ }^{82}$ also studied the possibility of using MMA-SPM 
NPs for the codelivery of all three antiretroviral drugs. They developed polymeric NPs that were also grafted with RMP-7 (surface functionalization performed by carbodiimide chemistry with poly[ethylene glycol] [PEG]-COOH to allow cross-linking with RMP-7) in order to promote BBB crossing by modulating endocytosis and TJ opening. This strategy was shown to be mildly effective in improving the permeability of considered drugs across another in vitro BBB model (monolayer of HBMECs regulated by human astrocytes in a dual-chamber setup). For example, drugpermeability coefficients were increased approximately 1.4-, 2.1- and 1.4-fold for stavudine, delavirdine, and saquinavir, respectively, when compared to values obtained for similar NPs not bearing RMP-7 at their surface. Finally, the impact of EMF exposure on the permeability of saquinavir across the BBB model, as mediated by drug incorporation in the same type of polymeric NPs and SLNs, was studied by Kuo and Kuo. ${ }^{83}$ Higher EMF frequency yielded larger permeability coefficients when both drug-loaded nanocarriers and free saquinavir were used, while square waves produced greater permeability than sine and triangle waves. Moreover, and also important to note, higher EMF power caused apoptosis of HBMECs, a fact that can impair the usefulness of this additional strategy due to toxicity issues.

Albumin is a matrix-forming macromolecule commonly used in the production of drug nanocarriers, mainly due to its advantageous biodegradability and low toxicity properties. Albumin NPs encapsulating zidovudine have been proposed for brain delivery of this polar nucleoside; ${ }^{77}$ alongside, surface modification of NPs with PEG was performed in order to reduce their rapid removal from blood circulation. The NP surface was further modified by anchoring Tf ligand with the purpose of enhancing CNS uptake. The biodistribution of zidovudine was studied in Wistar rats after intravenous administration of unmodified and Tf-modified PEG-albumin NPs with an equivalent dose of $54.4 \mathrm{mg}$ drug $/ \mathrm{kg}$ of body weight. Experimental results showed significant enhancement of brain drug localization for Tf-PEG-albumin NPs compared to PEG-albumin NPs $(21.1 \% \pm 1.8 \%$ versus $9.3 \% \pm 0.9 \%$, respectively, of the total drug recovered at 4 hours postadministration). This study confirmed the potential use of Tf modification of nanocarriers in order to enhance brain drug targeting for HIV therapy.

In order to target macrophages, either at the CNS or the mononuclear phagocyte system, Kaur et $\mathrm{al}^{81}$ developed didanosine-loaded gelatin NPs with mannan coating. These macromolecular (gelatin) NPs were produced by double desolvation, and in order to coat them, they were incubated with a mannan solution. Besides providing sustained drug release, this nanosystem improved in vitro drug uptake by macrophages, with a fivefold increase in cell-associated levels after 2 hours' incubation compared with didanosine in solution. Fluorescence microscopy also confirmed NP cell internalization. Further, didanosine levels in the brain were increased by 12.4-fold upon subcutaneous administration of mannan-coated NPs compared to didanosine in solution (Figure 2). ${ }^{81}$ Also, brain accumulation of didanosine was higher than when plain NPs (ie, without mannan surface modification) were used. These results seem to support the positive influence of mannose residues in the ability of NPs to reach the CNS, probably by a monocyte/macrophagemediated mechanism.

Poly(L-lactic acid) NPs conjugated with Tat peptide were also studied in order to increase the transport of ritonavir across the $\mathrm{BBB}$ and into the CNS. ${ }^{73,74}$ This strategy was envisioned in order to bypass the known efflux action of P-gp on this particular PI. NPs were produced by an emulsionsolvent evaporation technique and Tat functionalization achieved by using an epoxy conjugation method, where the NP surface is activated by epoxy compound followed by Tat conjugation. NPs were shown to be effective in vitro in inhibiting HIV-1 infection of monocyte-derived macrophages through the reduction of cytopathic effects, HIV-1 p24 protein secretion, and production of progeny virions. ${ }^{74}$ Also, P-gp intact (wild-type) mice were injected via the tail vein with either ritonavir-loaded NPs or the drug in solution (45 mg/kg ritonavir in both cases). ${ }^{73}$ Obtained data showed an increase in the ritonavir brain parenchyma:capillary ratio over time (Figure 3), without disruption of BBB integrity, in animals that received drug-loaded Tat-conjugated NPs; after 2 weeks, brain drug level with Tat-conjugated NPs was 800 -fold higher than that with the drug in solution, and about sevenfold higher than unconjugated NPs. Rao et al proposed that Tat-conjugated NPs were transported to the parenchyma without influencing the integrity of the BBB, which suggests that this transport could occur due to transcytosis across the endothelium of the brain vasculature. Moreover, these NPs maintained potentially therapeutic drug levels in the brain for a sustained period (14 days) with a single-dose intravenous administration. ${ }^{73}$ Therefore, Tat-NPs may constitute an effective way of delivering anti-HIV-1 drugs to the CNS.

In another approach, Mahajan et $\mathrm{al}^{95}$ proposed the use of Tf-conjugated quantum rods (QRs; average length and diameter of approximately $25 \mathrm{~nm}$ and $5 \mathrm{~nm}$, respectively, as assessed by transmission electron microscopy) in order to enhance the brain delivery of saquinavir. QRs were composed 


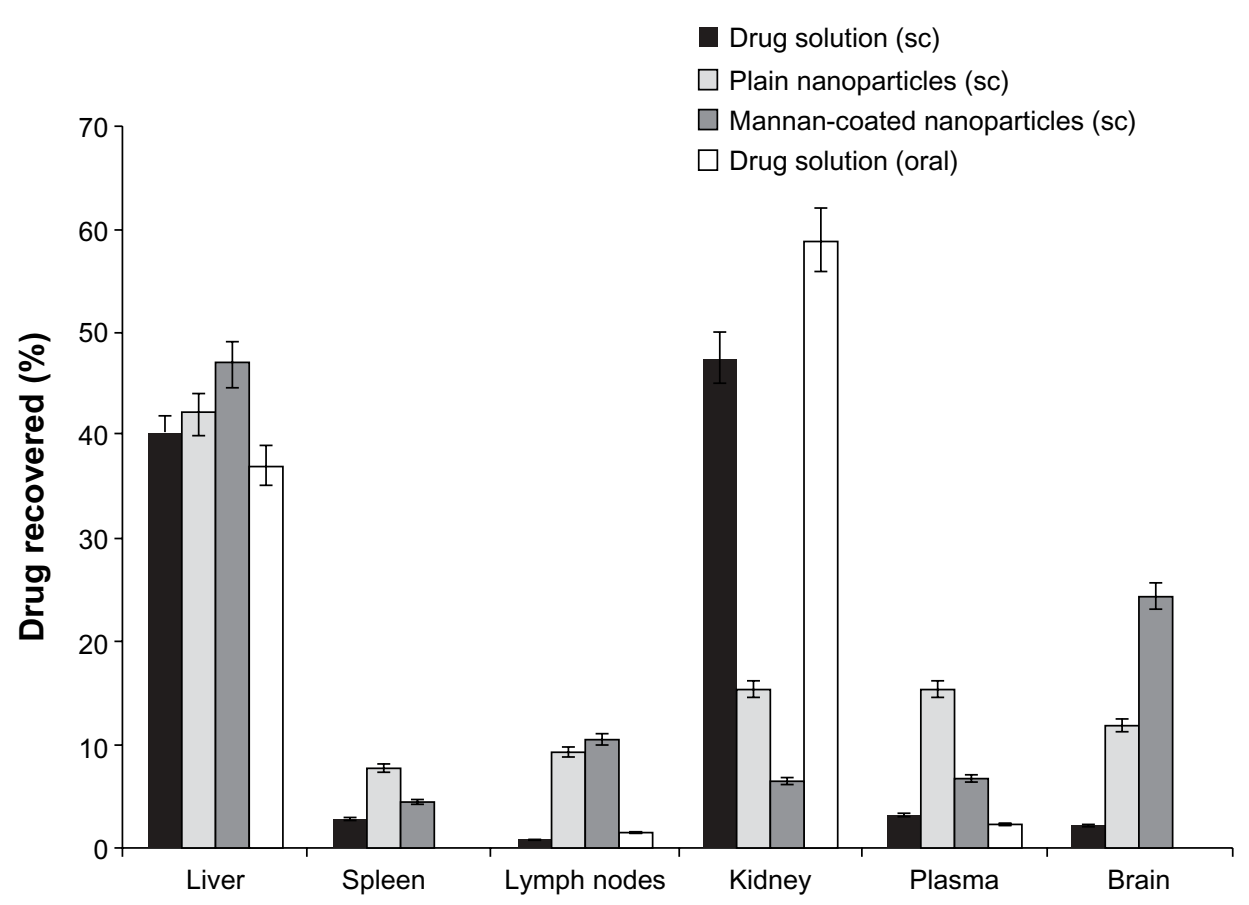

Figure 2 Tissue distribution of didanosine (after 12 hours of administration) delivered as mannan-coated nanoparticles, plain nanoparticles, and in solution (mean \pm standard deviation, $\mathrm{n}=3$ ).

Notes: Reproduced from Acta Pharmaceutica, 2008, vol 58, No I, page 7I. Kaur A, Jain S, Tiwary AK. Mannan-coated gelatin nanoparticles for sustained and targeted delivery of didanosine: in vitro and in vivo evaluation. ${ }^{81}$

Abbreviation: SC, subcutaneous.
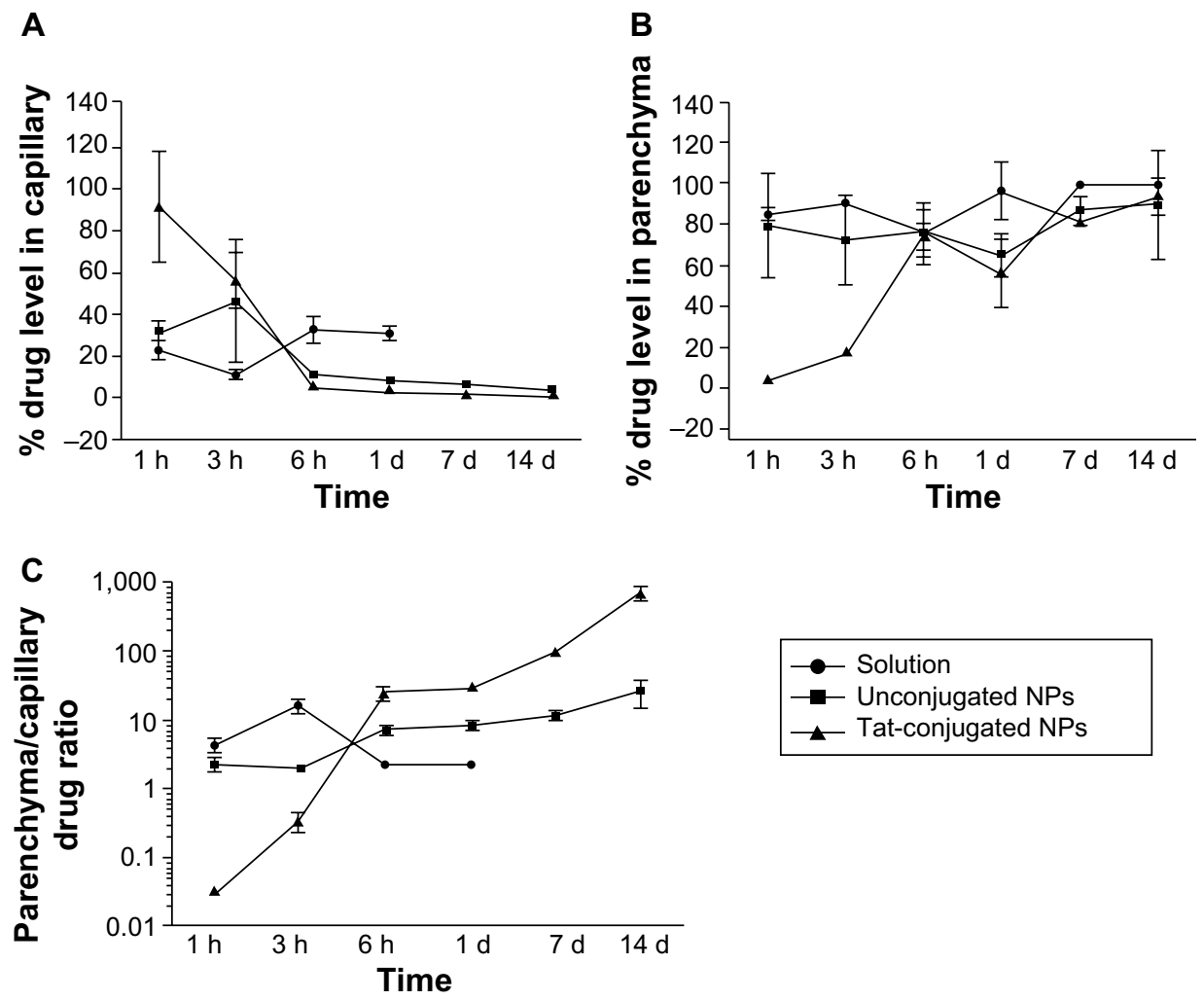

Figure 3 Changes in the fraction of ritonavir distribution between brain capillaries and parenchyma over time following intravenous drug administration.

Notes: The fractions of the total brain drug present in (A) brain capillaries and (B) brain parenchyma, and (C) the ratio of drug distribution between the brain parenchyma/ capillaries in different groups is presented. The capillary drug levels in the solution group were undetectable beyond I day after administration. Data are represented as means \pm standard error of the mean $(n=4)$. $* P<0.05$ (Student's $t$-test) between Tat-conjugated and unconjugated nanoparticles (NPs). Reprinted from Biomaterials, $2008 ; 29$. Rao KS, Reddy MK, Horning JL, Labhasetwar V TAT-conjugated nanoparticles for the CNS delivery of anti-HIV drugs. 4429-4438. () (2008) with permission Elsevier. ${ }^{73}$ Abbreviations: h, hour(s); d, day(s); Tat, transactivator of transcription. 
of a thin zinc sulfide layer over a cadmium/selenium core nanocrystal and coated with mercaptosuccinic acid; saquinavir and Tf were further conjugated to surface carboxyl groups by carbodiimide chemistry and stabilized by mixing with poloxamer 407 (Figure 4). The nanocarrier was tested in an in vitro BBB model comprising primary cultures of HBMECs and normal human astrocytes in a double-chamber setup. An HBMEC monolayer was grown in the apical side of a porous polyethylene terephthalate membrane, while the basolateral side was covered with an astrocyte monolayer. Additionally, HIV-1-infected peripheral blood mononuclear cells (PBMCs) were placed in the receptor chamber in order to evaluate the antiretroviral efficacy of different treatments. Results showed that QRs did not affect the integrity of the $\mathrm{BBB}$, and presented reduced cytotoxicity of up to $40 \mathrm{nM}$ concentration in saquinavir; also, functionalization with $\mathrm{Tf}$ led to enhanced uptake of QRs by HBMECs and PBMCs compared to nonfunctionalized QRs. Moreover, when the above BBB model was used, a significant decrease of HIV-1 replication in PBMCs was observed for Tf-saquinavir QRs compared to both the free drug and non-Tf-functionalized saquinavir QRs. These observations seem to support the view that the proposed QRs may provide an efficient way to promote the transport of saquinavir across this barrier and reduce the viral load at the CNS. Nevertheless, the mechanism of release of conjugated drugs from the carrier is not clear, even though the authors claim that degradation of the nanosystem as a whole is involved. ${ }^{96}$ Comparable results were also reported for Tf-conjugated QRs carrying amprenavir. ${ }^{96}$ In addition, these QRs present the ability to be used in realtime tracking of particle crossing through the in vitro BBB model, as well as a potential use in diagnostic imaging, due to their intrinsic optical properties. ${ }^{97}$ Of further note, nano-

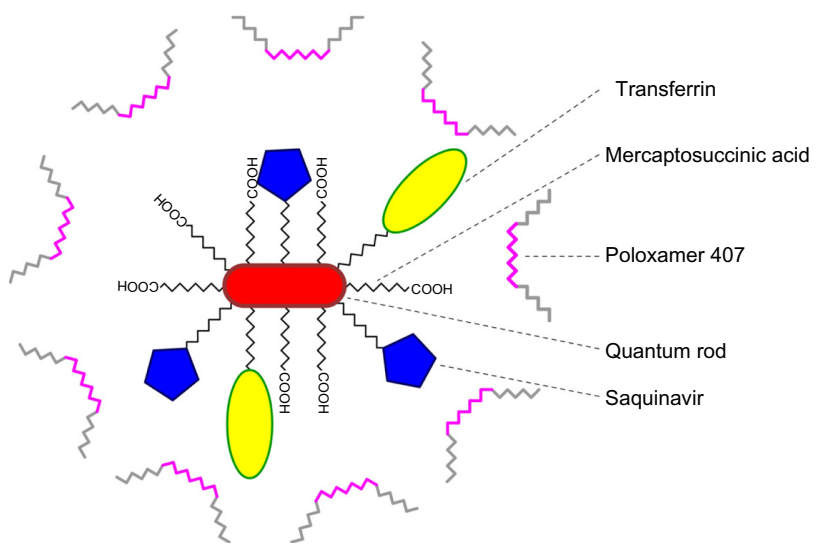

Figure 4 Schematic representation of the quantum rod-based nanosystem for the delivery of saquinavir.

Note: Data from Mahajan et al..$^{95}$ plexes have also been obtained by the same group by using quantum dots (QDs) produced with the same materials and presenting a similar structure as QRs (in this case, Tf was not conjugated and poly[diallyldimethylammonium chloride] was used to provide a positive surface charge to QDs) and siRNA targeting the expression of matrix-degrading metalloproteinase type- $9 .{ }^{98}$ This protein plays a significant role in disrupting the BBB, and its expression is triggered by HIV-1 infection of the CNS. Results using the aforementioned in vitro BBB model showed that proposed siRNA-QD nanoplexes were able to silence gene expression in HBMECs and thus maintain the integrity of the membrane. Although not directly targeting the virus, this delivery system may provide an interesting auxiliary approach in preventing continuous viral invasion of the CNS.

\section{Antiretroviral drug nanosuspensions for CNS delivery}

Added to the previous examples of different nanocarriers, size reduction to the low-micrometer/nanometer scale of antiretroviral drugs, and their eventual surface modification, may also be an interesting strategy of enhancing drug delivery to the CNS. For example, Shegokar and Singh ${ }^{99}$ prepared nanosuspensions of nevirapine by high-pressure homogenization and proceeded with surface modification with serum albumin, PEG 1000, or dextran 60 by physical adsorption in solution (final diameters around $500 \mathrm{~nm}$ ). When tested in vivo in a rat model, particles modified with albumin presented the ability to accumulate in the brain compared to nevirapine in solution or other drug nanosuspensions (in the previous cases, no drug was detected at this anatomical site). The area under the curve in the brain $\left(\mathrm{AUC}_{\text {brain }}\right) / \mathrm{AUC}_{\text {blood }}$ ratio of albuminmodified nanosuspensions was 9.33. Although no specific mechanistic explanation for the observed enhancement of brain drug levels was provided by the authors, increased macrophage uptake of albumin-modified nevirapine particles over other formulations may be at least partially associated with differential patterns of adsorbed proteins at the particle surface. ${ }^{99,100} \mathrm{~A}$ recent study by Dash et al ${ }^{101}$ further demonstrated the potential of using nanosuspensions of atazanavir and ritonavir obtained by high-pressure homogenization and using poloxamer 188 as a stabilizer for neuroprotection in a humanized HIV-infected animal model (nonobese diabetic/severe combined immunodeficiency- $\gamma_{c}{ }^{\text {null }}$ mice). Weekly intravenous administration of nanosuspensions allowed the eliciting of neuroprotective responses (as assessed by reduced neuronal, synaptic, and astrocyte damage), alongside the reduction of viral loads and maintenance 
of $\mathrm{CD}^{+}$cells in peripheral blood. A further study by the same group using the same in vivo model confirmed these observations and demonstrated its relation with tissue and blood serum levels of atazanavir, ritonavir, and efavirenz when administered as nanosuspensions. ${ }^{102}$ Again, the role of monocytes/macrophages on the enhanced CNS delivery of the antiretroviral drugs was emphasized.

Indeed, monocytes/macrophages may be an ideal physiological "shuttle" for brain delivery of antiretroviral drugs, due to their phagocytic nature, which allows extensive uptake (depending on coating, size, shape, and charge) and sustained release (for days to weeks) of different antiretroviral drug particles. ${ }^{103-105}$ Additionally, these immune cells possess the ability to readily migrate across the $\mathrm{BBB}$ or at least transfer their drug content to endothelial cells at the BBB, as recently shown in vitro (Figure 5). ${ }^{106}$ The previous possibilities have been systematically explored by investigators at the Nebraska Medical Center, Omaha, NE, USA. ${ }^{103-109}$ Drug incorporation into cells was performed by simply incubating cells with particles in culture media for 8-12 hours, followed by cell washing and removal of free drug. Researchers observed that nanoformulated indinavir could be delivered into the brain of an HIV-1 encephalitis rodent model up to around 20-fold higher levels when incorporated into bone marrow-derived macrophages after a single dose administered intravenously. ${ }^{109}$ Moreover, treatment with this cell-based nanoformulation was able to release indinavir in a continuous fashion over 2 weeks and reduce HIV replication in brain regions presenting signs of encephalitis. This may well be associated with the role played by used drug-carrier cells in the inflammatory response at HIV-replication sites, thus providing a natural targeting mechanism for antiretroviral drugs. One important aspect of using monocytes/macrophages for the delivery of nanosized antiretroviral drugs to the CNS is related to the potential of drug-induced toxicity to cell carriers. Even if toxicity to human monocytes/macrophages and HBMECs has been shown to be generally low in vitro when cells were incubated with different ritonavir, indinavir, and efavirenz particles up to concentrations of $100 \mu \mathrm{M}$, a considerable decrease in cell viability was observed for levels of $500 \mu \mathrm{M} .{ }^{110}$ Moreover, toxicity (viability, release of inflammatory mediators, and reduction of the transendothelial electrical resistance of primary HBMEC monolayers) was dependent on the combination of used antiretroviral drugs and nanosuspension parameters, namely surface charge, surfactants (used as stabilizers), and particle shape and size. Although these concentrations may not be observed in vivo, ${ }^{104,106,109}$ special
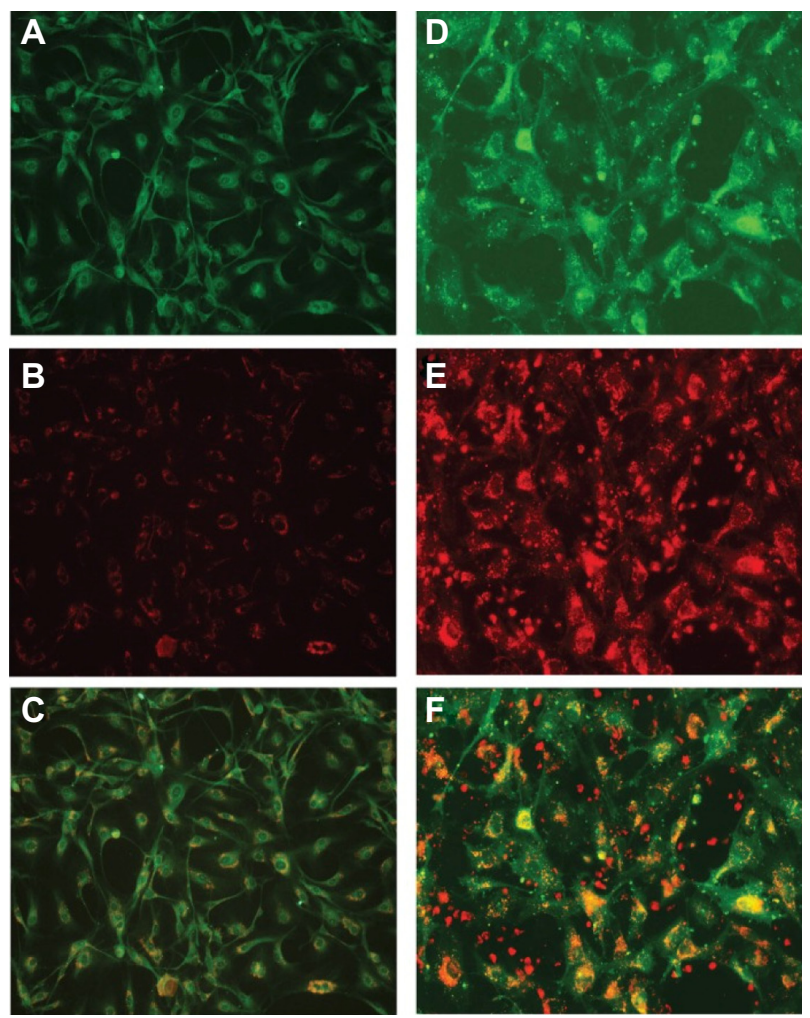

Figure $\mathbf{5}$ Uptake of antiretroviral drugs formulated as nanosuspensions by human brain microvascular endothelial cells (HBMECs) following endothelial mononuclear phagocyte cocultivation.

Notes: (A-C) Primary HBMECs were exposed for 2 hours to conditioned media from monocyte-derived macrophages loaded with rhodamine-labeled efavirenz nanosuspension ( $300 \mathrm{~nm}$ and $+7.4 \mathrm{mV}$; obtained by sonication and using poly[lactideco-glycolide], poly[vinyl alcohol] and cetrimide as stabilizers); HBMECs were then washed thrice with phosphate-buffered saline to remove macrophage-conditioned media, and analyzed by fluorescence microscopy. (B and $\mathbf{C}$ ) Endothelial cell uptake of nanosuspensions (orange-yellow) released by monocyte-derived macrophages. Staining with the endothelium-specific marker von Willebrand factor (green, A) confirmed the endothelial nature of primary HBMECs. (D-F) Primary HBMECs labeled with Vybrant I, I'-dioctadecyl-3,3,3',3'-tetramethylindodicarbocyanine perchlorate (green, D) were cocultured for 2 hours with monocytes loaded with rhodamine-labeled ritonavir $(6 / 3 \mathrm{~nm}$ and $-26.7 \mathrm{mV})$; obtained by high-pressure homogenization and using poloxamer 188 and $\mathrm{N}$-(carbonyl-methoxy-polyethylene glycol 2000)-1,2-sn-glycero-3-phosphoethanolamine as stabilizers, washed to remove monocytes, and analyzed by fluorescence microscopy. (E and $\mathbf{F}$ ) Endothelial cell uptake of nanosuspensions (orange-yellow) during endothelial monocyte cocultivation. Copyright @2012. Dove Medical Press. Reproduced from Kanmogne GD, Singh S, Roy U, et al. Mononuclear phagocyte intercellular crosstalk facilitates transmission of cell-targeted nanoformulated antiretroviral drugs to human brain endothelial cells. Int J Nanomedicine. 2012;7:2373-2388. ${ }^{106}$

attention should be paid to these issues in order to reduce the potential onset of safety issues.

\section{Conclusion and remaining challenges}

Tremendous progress has been achieved in the field of HIV/AIDS management over the last few decades. The development of different antiretroviral drugs alongside the introduction of cART allowed a shift of the infection from rapidly progressive to a chronic disease. Even so, a cure is 
still elusive, and refinement of current therapy is required. Restriction of CNS infection by HIV and the management of its consequences are limited by poor antiretroviral pharmacokinetics, which are often associated with poor compound permeation of the BBB. Nanotechnology-based solutions have shown the potential to provide efficient and safe tools to circumvent these problems, and in particular solid drug nanocarriers and drug nanosuspensions may be preferential, due to their ability to prolong systemic circulation, provide anatomical and/or cell targeting, increase crossing and reduce drug efflux at the BBB, and enhance intracellular drug levels at HIV-target cells. Moreover, the inherent toxicity of many drugs currently being used in cART may be reduced.

Despite the work developed so far, important questions remain. One limitation of current strategies is related to the need for systemic delivery of NPs in order to allow them to reach the $\mathrm{BBB}$ and deliver drugs into the CNS. Interestingly, we are not aware of any study considering the delivery of nanosystems by alternative routes with the purpose of antiretroviral therapy. The intranasal delivery of nanomedicines may be an interesting option due to the direct nose-to-brain transport, which bypasses the BBB. ${ }^{111}$ Another issue is related to the accumulation of antiretroviral drugs and even nanocarriers at the CNS and their potential neurotoxicity, particularly when long-term regimens are needed. The biodistribution patterns of NPs once in the CNS, alongside their long-term fate, also require additional understanding. ${ }^{112}$ Indeed, the field of nanotoxicology is growing exponentially, and the potentially deleterious effects of nanomedicines crossing the BBB require further clarification. ${ }^{113}$ Finally, the true impact and the possibility of viral eradication with the use of nanotechnology-based cART needs to be assessed in vivo, while optimization of dosages and schedules is needed. Answering these and other questions adequately is mandatory in order to open the way to the translation of such therapeutics to exploratory human clinical trials. The different investigations so far conducted and reviewed in this manuscript, alongside the prolific work being developed in the field, assure that these issues will be addressed in coming years.

\section{Acknowledgments}

Maria João Gomes and José das Neves gratefully acknowledge Fundação para a Ciência e a Tecnologia (FCT), Portugal for financial support (grants SFRH/BD/90404/2012 and SFRH/ BPD/92934/2013, respectively). This work was financed by a grant from FCT (reference VIH/SAU/0021/2011) and by the European Regional Development Fund (ERDF) through the Programa Operacional Factores de Competitividade (COMPETE), by Portuguese funds through FCT in the framework of the project PEst-C/SAU/LA0002/2013, and cofinanced by North Portugal Regional Operational Programme (ON.2 - O Novo Norte) in the framework of project SAESCTN-PIIC\&DT/2011, under the National Strategic Reference Framework (NSRF).

\section{Disclosure}

The authors report no conflicts of interest in this work.

\section{References}

1. Joint United Nations Programme on HIV/AIDS. 2013 UNAIDS Report on the Global AIDS Epidemic. Geneva: UNAIDS; 2013. Available from: http://www.unaids.org/en/resources/documents/2013/name,85053,en. asp. Accessed January 23, 2014.

2. Beard J, Feeley F, Rosen S. Economic and quality of life outcomes of antiretroviral therapy for HIV/AIDS in developing countries: a systematic literature review. AIDS Care. 2009;21:1343-1356.

3. Bor J, Herbst AJ, Newell ML, Barnighausen T. Increases in adult life expectancy in rural South Africa: valuing the scale-up of HIV treatment. Science. 2013;339:961-965.

4. Volberding PA, Deeks SG. Antiretroviral therapy and management of HIV infection. Lancet. 2010;376:49-62.

5. Letendre S, Marquie-Beck J, Capparelli E, et al. Validation of the CNS penetration-effectiveness rank for quantifying antiretroviral penetration into the central nervous system. Arch Neurol. 2008;65:65-70.

6. Gimenez F, Fernandez C, Mabondzo A. Transport of HIV protease inhibitors through the blood-brain barrier and interactions with the efflux proteins, P-glycoprotein and multidrug resistance proteins. J Acquir Immune Defic Syndr. 2004;36:649-658.

7. Smurzynski M, Wu K, Letendre S, et al. Effects of central nervous system antiretroviral penetration on cognitive functioning in the ALLRT cohort. AIDS. 2011;25:357-365.

8. Destache CJ. Brain as an HIV sequestered site: use of nanoparticles as a therapeutic option. Prog Brain Res. 2009;180:225-233.

9. Nowacek A, Gendelman HE. NanoART, neuroAIDS and CNS drug delivery. Nanomedicine (Lond). 2009;4:557-574.

10. Rao KS, Ghorpade A, Labhasetwar V. Targeting anti-HIV drugs to the CNS. Expert Opin Drug Deliv. 2009;6:771-784.

11. das Neves J, Amiji MM, Bahia MF, Sarmento B. Nanotechnology-based systems for the treatment and prevention of HIV/AIDS. Adv Drug Deliv Rev. 2010;62:458-477.

12. Wong HL, Chattopadhyay N, Wu XY, Bendayan R. Nanotechnology applications for improved delivery of antiretroviral drugs to the brain. Adv Drug Deliv Rev. 2010;62:503-517.

13. Mahajan SD, Aalinkeel R, Law WC, et al. Anti-HIV-1 nanotherapeutics: promises and challenges for the future. Int J Nanomedicine. 2012;7: 5301-5314.

14. Dutta T, Agashe HB, Garg M, Balakrishnan P, Kabra M, Jain NK. Poly(propyleneimine) dendrimer based nanocontainers for targeting of efavirenz to human monocytes/macrophages in vitro. J Drug Target. 2007;15:89-98.

15. Vyas TK, Shahiwala A, Amiji MM. Improved oral bioavailability and brain transport of saquinavir upon administration in novel nanoemulsion formulations. Int J Pharm. 2008;347:93-101.

16. Prabhakar K, Afzal SM, Kumar PU, Rajanna A, Kishan V. Brain delivery of transferrin coupled indinavir submicron lipid emulsions pharmacokinetics and tissue distribution. Colloids Surf B Biointerfaces. 2011;86:305-313.

17. Saiyed ZM, Gandhi NH, Nair MP. Magnetic nanoformulation of azidothymidine 5 -triphosphate for targeted delivery across the blood-brain barrier. Int J Nanomedicine. 2010;5:157-166. 
18. Chiappetta DA, Hocht C, Opezzo JA, Sosnik A. Intranasal administration of antiretroviral-loaded micelles for anatomical targeting to the brain in HIV. Nanomedicine (Lond). 2013;8:223-237.

19. Gerson T, Makarov E, Senanayake TH, Gorantla S, Poluektova LY, Vinogradov SV. Nano-NRTIs demonstrate low neurotoxicity and high antiviral activity against HIV infection in the brain. Nanomedicine. 2014;10:177-185.

20. González-Scarano F, Martín-García J. The neuropathogenesis of AIDS. Nat Rev Immunol. 2005;5:69-81.

21. Boissé L, Gill MJ, Power C. HIV infection of the central nervous system: clinical features and neuropathogenesis. Neurol Clin. 2008;26: 799-819.

22. Letendre SL, Ellis RJ, Ances BM, McCutchan JA. Neurologic complications of HIV disease and their treatment. Top HIV Med. 2010;18: 45-55.

23. Heaton RK, Franklin DR, Ellis RJ, et al. HIV-associated neurocognitive disorders before and during the era of combination antiretroviral therapy: differences in rates, nature, and predictors. J Neurovirol. 2011;17:3-16.

24. Brew BJ. Evidence for a change in AIDS dementia complex in the era of highly active antiretroviral therapy and the possibility of new forms of AIDS dementia complex. AIDS. 2004;18 Supp1 1:S75-S78.

25. Valcour V, Sithinamsuwan P, Letendre S, Ances B. Pathogenesis of HIV in the central nervous system. Curr HIV/AIDS Rep. 2011;8:54-61.

26. Blankson JN, Persaud D, Siliciano RF. The challenge of viral reservoirs in HIV-1 infection. Annu Rev Med. 2002;53:557-593.

27. Blum MR, Liao SH, Good SS, de Miranda P. Pharmacokinetics and bioavailability of zidovudine in humans. Am J Med. 1988;85: 189-194.

28. Shelton MJ, O’Donnell AM, Morse GD. Didanosine. Ann Pharmacother. 1992;26:660-670.

29. Haworth SJ, Christofalo B, Anderson RD, Dunkle LM. A single-dose study to assess the penetration of stavudine into human cerebrospinal fluid in adults. J Acquir Immune Defic Syndr Hum Retrovirol. 1998;17: 235-238.

30. Antinori A, Perno CF, Giancola ML, et al. Efficacy of cerebrospinal fluid (CSF)-penetrating antiretroviral drugs against HIV in the neurological compartment: different patterns of phenotypic resistance in CSF and plasma. Clin Infect Dis. 2005;41:1787-1793.

31. van Leeuwen R, Katlama C, Kitchen V, et al. Evaluation of safety and efficacy of 3TC (lamivudine) in patients with asymptomatic or mildly symptomatic human immunodeficiency virus infection: a phase I/II study. J Infect Dis. 1995;171:1166-1171.

32. Foster RH, Faulds D. Abacavir. Drugs. 1998;55:729-736.

33. Calcagno A, Bonora S, Simiele M, et al. Tenofovir and emtricitabine cerebrospinal fluid-to-plasma ratios correlate to the extent of blood-brain barrier damage. AIDS. 2011;25:1437-1439.

34. Best BM, Letendre SL, Koopmans P, et al. Low cerebrospinal fluid concentrations of the nucleotide HIV reverse transcriptase inhibitor, tenofovir. J Acquir Immune Defic Syndr. 2012;59:376-381.

35. Smith PF, DiCenzo R, Morse GD. Clinical pharmacokinetics of non-nucleoside reverse transcriptase inhibitors. Clin Pharmacokinet. 2001;40:893-905.

36. Tiraboschi JM, Niubo J, Vila A, Perez-Pujol S, Podzamczer D. Etravirine concentrations in CSF in HIV-infected patients. J Antimicrob Chemother. 2012;67:1446-1448.

37. Vella S, Floridia M. Saquinavir: clinical pharmacology and efficacy. Clin Pharmacokinet. 1998;34:189-201.

38. Kravcik S, Gallicano K, Roth V, et al. Cerebrospinal fluid HIV RNA and drug levels with combination ritonavir and saquinavir. J Acquir Immune Defic Syndr. 1999;21:371-375.

39. Aweeka F, Jayewardene A, Staprans S, et al. Failure to detect nelfinavir in the cerebrospinal fluid of HIV-1-infected patients with and without AIDS dementia complex. J Acquir Immune Defic Syndr Hum Retrovirol. 1999;20:39-43.

40. Best BM, Letendre SL, Brigid E, et al. Low atazanavir concentrations in cerebrospinal fluid. AIDS. 2009;23:83-87.
41. Croteau D, Letendre S, Best BM, et al. Therapeutic amprenavir concentrations in cerebrospinal fluid. Antimicrob Agents Chemother. 2012;56: 1985-1989.

42. Yilmaz A, Izadkhashti A, Price RW, et al. Darunavir concentrations in cerebrospinal fluid and blood in HIV-1-infected individuals. AIDS Res Hum Retroviruses. 2009;25:457-461.

43. Price RW, Parham R, Kroll JL, et al. Enfuvirtide cerebrospinal fluid (CSF) pharmacokinetics and potential use in defining CSF HIV-1 origin. Antivir Ther. 2008;13:369-374.

44. Croteau D, Best BM, Letendre S, et al. Lower than expected maraviroc concentrations in cerebrospinal fluid exceed the wild-type $\mathrm{CC}$ chemokine receptor 5-tropic HIV-1 50\% inhibitory concentration. AIDS. 2012;26:890-893.

45. Yilmaz A, Gisslen M, Spudich S, et al. Raltegravir cerebrospinal fluid concentrations in HIV-1 infection. PLoS One. 2009;4:e6877.

46. Letendre S, Mills A, Tashima K, et al. Distribution and antiviral activity in cerebrospinal fluid (CSF) of the integrase inhibitor, dolutegravir (DTG): ING116070 week 16 results [abstract 178LB]. 20th Conference on Retroviruses and Opportunistic Infections; March 3-6, 2013; Atlanta, GA.

47. Berger JR, Avison M. The blood brain barrier in HIV infection. Front Biosci. 2004;9:2680-2685.

48. McGee B, Smith N, Aweeka F. HIV pharmacology: barriers to the eradication of HIV from the CNS. HIV Clin Trials. 2006;7:142-153.

49. Kumar AM, Borodowsky I, Fernandez B, Gonzalez L, Kumar M. Human immunodeficiency virus type 1 RNA Levels in different regions of human brain: quantification using real-time reverse transcriptasepolymerase chain reaction. J Neurovirol. 2007;13:210-224.

50. Abbott NJ, Patabendige AA, Dolman DE, Yusof SR, Begley DJ. Structure and function of the blood-brain barrier. Neurobiol Dis. 2010;37:13-25.

51. Santaguida S, Janigro D, Hossain M, Oby E, Rapp E, Cucullo L. Side by side comparison between dynamic versus static models of blood-brain barrier in vitro: a permeability study. Brain Res. 2006;1109:1-13.

52. Bhaskar S, Tian F, Stoeger T, et al. Multifunctional nanocarriers for diagnostics, drug delivery and targeted treatment across blood-brain barrier: perspectives on tracking and neuroimaging. Part Fibre Toxicol. 2010;7:3.

53. Cecchelli R, Berezowski V, Lundquist S, et al. Modelling of the bloodbrain barrier in drug discovery and development. Nat Rev Drug Discov. 2007;6:650-661.

54. Murthy SK. Nanoparticles in modern medicine: state of the art and future challenges. Int J Nanomedicine. 2007;2:129-141.

55. Hervé F, Ghinea N, Scherrmann JM. CNS delivery via adsorptive transcytosis. AAPS J. 2008;10:455-472.

56. Nagpal K, Singh SK, Mishra DN. Drug targeting to brain: a systematic approach to study the factors, parameters and approaches for prediction of permeability of drugs across BBB. Expert Opin Drug Deliv. 2013;10(7):927-955.

57. Pardridge WM. Blood-brain barrier drug targeting: the future of brain drug development. Mol Interv. 2003;3:90.

58. Dean M, Hamon Y, Chimini G. The human ATP-binding cassette (ABC) transporter superfamily. J Lipid Res. 2001;42:1007-1017.

59. Begley DJ. ABC transporters and the blood-brain barrier. Curr Pharm Design. 2004;10:1295-1312.

60. Dauchy S, Dutheil F, Weaver RJ, et al. ABC transporters, cytochromes P450 and their main transcription factors: expression at the human blood-brain barrier. J Neurochem. 2008;107:1518-1528.

61. De Boer A, Gaillard P. Drug targeting to the brain. Annu Rev Pharmacol Toxicol. 2007;47:323-355.

62. Hartz A, Bauer B. Regulation of ABC transporters at the blood-brain barrier: new targets for CNS therapy. Mol Interv. 2010;10:293.

63. Juillerat-Jeanneret L. The targeted delivery of cancer drugs across the blood-brain barrier: chemical modifications of drugs or drugnanoparticles? Drug Discov Today. 2008;13:1099-1106.

64. Jain KK. The Handbook of Nanomedicine. 2nd ed. New York: Springer; 2012 . 
65. Jain K. Role of nanotechnology in developing new therapies for diseases of the nervous system. Nanomedicine (Lond). 2006;1:9-12.

66. Lockman P, Mumper R, Khan M, Allen D. Nanoparticle technology for drug delivery across the blood-brain barrier. Drug Dev Ind Pharm. 2002;28:1-13.

67. Gessner A, Lieske A, Paulke B, Müller R. Influence of surface charge density on protein adsorption on polymeric nanoparticles: analysis by two-dimensional electrophoresis. Eur J Pharm Biopharm. 2002;54: $165-170$.

68. Wilson B, Samanta MK, Santhi K, Kumar KP, Paramakrishnan N, Suresh B. Targeted delivery of tacrine into the brain with polysorbate 80-coated poly(n-butylcyanoacrylate) nanoparticles. Eur J Pharm Biopharm. 2008;70:75-84.

69. Tahara K, Miyazaki Y, Kawashima Y, Kreuter J, Yamamoto H. Brain targeting with surface-modified poly(D,L-lactic-co-glycolic acid) nanoparticles delivered via carotid artery administration. Eur J Pharm Biopharm. 2011;77:84-88.

70. Göppert TM, Müller RH. Polysorbate-stabilized solid lipid nanoparticles as colloidal carriers for intravenous targeting of drugs to the brain: comparison of plasma protein adsorption patterns. J Drug Target. 2005;13:179-187.

71. Kreuter J, Shamenkov D, Petrov V, et al. Apolipoprotein-mediated transport of nanoparticle-bound drugs across the blood-brain barrier. J Drug Target. 2002;10:317-325.

72. Lindgren M, Langel U. Classes and prediction of cell-penetrating peptides. Methods Mol Biol. 2011;683:3-19.

73. Rao KS, Reddy MK, Horning JL, Labhasetwar V. TAT-conjugated nanoparticles for the CNS delivery of anti-HIV drugs. Biomaterials. 2008;29:4429-4438.

74. Borgmann K, Rao KS, Labhasetwar V, Ghorpade A. Efficacy of Tat-conjugated ritonavir-loaded nanoparticles in reducing HIV-1 replication in monocyte-derived macrophages and cytocompatibility with macrophages and human neurons. AIDS Res Hum Retroviruses. 2011;27:853-862.

75. Gaillard PJ, Appeldoorn CC, Rip J, et al. Enhanced brain delivery of liposomal methylprednisolone improved therapeutic efficacy in a model of neuroinflammation. J Control Release. 2012;164: 364-369.

76. Moos T, Morgan EH. Transferrin and transferrin receptor function in brain barrier systems. Cell Mol Neurobiol. 2000;20:77-95.

77. Mishra V, Mahor S, Rawat A, et al. Targeted brain delivery of AZT via transferrin anchored pegylated albumin nanoparticles. J Drug Target. 2006;14:45-53.

78. Gabathuler R. Approaches to transport therapeutic drugs across the blood-brain barrier to treat brain diseases. Neurobiol Dis. 2010;37: 48-57.

79. Vance JE, Hayashi H. Formation and function of apolipoprotein E-containing lipoproteins in the nervous system. Biochim Biophys Acta. 2010;1801:806-818.

80. Dehouck B, Fenart L, Dehouck MP, Pierce A, Torpier G, Cecchelli R. A new function for the LDL receptor: transcytosis of LDL across the blood-brain barrier. J Cell Biol. 1997;138:877-889.

81. Kaur A, Jain S, Tiwary AK. Mannan-coated gelatin nanoparticles for sustained and targeted delivery of didanosine: in vitro and in vivo evaluation. Acta Pharm. 2008;58:61-74.

82. Kuo YC, Lee CL. Methylmethacrylate-sulfopropylmethacrylate nanoparticles with surface RMP-7 for targeting delivery of antiretroviral drugs across the blood-brain barrier. Colloids Surf B Biointerfaces. 2012;90:75-82.

83. Kuo YC, Kuo CY. Electromagnetic interference in the permeability of saquinavir across the blood-brain barrier using nanoparticulate carriers. Int J Pharm. 2008;351:271-281.

84. Bauer B, Hartz AMS, Fricker G, Miller DS. Modulation of p-glycoprotein transport function at the blood-brain barrier. Exp Biol Med (Maywood). 2005;230:118-127.

85. Begley D. ABC transporters and the blood-brain barrier. Curr Pharm Des. 2004;10:1295-1312.
86. Schinkel AH, Wagenaar E, Mol CA, van Deemter L. P-glycoprotein in the blood-brain barrier of mice influences the brain penetration and pharmacological activity of many drugs. J Clin Investig. 1996;97:2517-2524.

87. Lage H. An overview of cancer multidrug resistance: a still unsolved problem. Cell Mol Life Sci. 2008;65:3145-3167.

88. Honjo K, Takahashi KA, Mazda O, et al. MDR1a/1b gene silencing enhances drug sensitivity in rat fibroblast-like synoviocytes. $J$ Gene Med. 2010;12:219-227.

89. Chen XP, Wang Q, Guan J, Huang ZY, Zhang WG, Zhang BX. Reversing multidrug resistance by RNA interference through the suppression of MDR1 gene in human hepatoma cells. World $J$ Gastroenterol. 2006;12:3332-3337.

90. Fuest C, Bankstahl M, Winter P, Helm M, Pekcec A, Potschka H. In vivo down-regulation of mouse brain capillary P-glycoprotein: a preliminary investigation. Neurosci Lett. 2009;464:47-51.

91. Joshy KS, Sharma CP. Blood Compatible nanostructured lipid carriers for the enhanced delivery of azidothymidine to brain. Adv Sci Lett. 2012;6:47-55

92. Chattopadhyay N, Zastre J, Wong HL, Wu XY, Bendayan R. Solid lipid nanoparticles enhance the delivery of the HIV protease inhibitor, atazanavir, by a human brain endothelial cell line. Pharm Res. 2008;25:2262-2271.

93. Markoutsa E, Pampalakis G, Niarakis A, et al. Uptake and permeability studies of BBB-targeting immunoliposomes using the hCMEC/D3 cell line. Eur J Pharm Biopharm. 2011;77:265-274.

94. Kuo YC, Su FL. Transport of stavudine, delavirdine, and saquinavir across the blood-brain barrier by polybutylcyanoacrylate, methylmethacrylate-sulfopropylmethacrylate, and solid lipid nanoparticles. Int J Pharm. 2007;340:143-152.

95. Mahajan SD, Roy I, Xu G, et al. Enhancing the delivery of anti retroviral drug "saquinavir" across the blood brain barrier using nanoparticles. Curr HIV Res. 2010;8:396-404.

96. Mahajan SD, Law WC, Aalinkeel R, et al. Nanoparticle-mediated targeted delivery of antiretrovirals to the brain. Methods Enzymol. 2012;509:41-60.

97. Xu G, Yong KT, Roy I, et al. Bioconjugated quantum rods as targeted probes for efficient transmigration across an in vitro blood-brain barrier. Bioconjug Chem. 2008;19:1179-1185.

98. Bonoiu A, Mahajan SD, Ye L, et al. MMP-9 gene silencing by a quantum dot-siRNA nanoplex delivery to maintain the integrity of the blood brain barrier. Brain Res. 2009;1282:142-155.

99. Shegokar R, Singh KK. Surface modified nevirapine nanosuspensions for viral reservoir targeting: In vitro and in vivo evaluation. Int $J$ Pharm. 2011;421:341-352.

100. Shegokar R, Jansch M, Singh KK, Müller RH. In vitro protein adsorption studies on nevirapine nanosuspensions for HIV/AIDS chemotherapy. Nanomedicine. 2011;7:333-340.

101. Dash PK, Gendelman HE, Roy U, et al. Long-acting nanoformulated antiretroviral therapy elicits potent antiretroviral and neuroprotective responses in HIV-1-infected humanized mice. AIDS. 2012;26: 2135-2144.

102. Roy U, McMillan J, Alnouti Y, et al. Pharmacodynamic and antiretroviral activities of combination nanoformulated antiretrovirals in HIV-1-infected human peripheral blood lymphocyte-reconstituted mice. J Infect Dis. 2012;206:1577-1588.

103. Nowacek AS, Miller RL, McMillan J, et al. NanoART synthesis, characterization, uptake, release and toxicology for human monocytemacrophage drug delivery. Nanomedicine (Lond). 2009;4:903-917.

104. Nowacek AS, McMillan J, Miller R, Anderson A, Rabinow B, Gendelman HE. Nanoformulated antiretroviral drug combinations extend drug release and antiretroviral responses in HIV-1-infected macrophages: implications for neuroAIDS therapeutics. J Neuroimmune Pharmacol. 2010;5:592-601.

105. Nowacek AS, Balkundi S, McMillan J, et al. Analyses of nanoformulated antiretroviral drug charge, size, shape and content for uptake, drug release and antiviral activities in human monocyte-derived macrophages. J Control Release. 2011;150:204-211. 
106. Kanmogne GD, Singh S, Roy U, et al. Mononuclear phagocyte intercellular crosstalk facilitates transmission of cell-targeted nanoformulated antiretroviral drugs to human brain endothelial cells. Int J Nanomedicine. 2012;7:2373-2388.

107. Dou H, Destache CJ, Morehead JR, et al. Development of a macrophage-based nanoparticle platform for antiretroviral drug delivery. Blood. 2006;108:2827-2835.

108. Dou H, Morehead J, Destache CJ, et al. Laboratory investigations for the morphologic, pharmacokinetic, and anti-retroviral properties of indinavir nanoparticles in human monocyte-derived macrophages. Virology. 2007;358:148-158.

109. Dou H, Grotepas CB, McMillan JM, et al. Macrophage delivery of nanoformulated antiretroviral drug to the brain in a murine model of neuroAIDS. J Immunol. 2009;183:661-669.
110. Bressani RF, Nowacek AS, Singh S, et al. Pharmacotoxicology of monocyte-macrophage nanoformulated antiretroviral drug uptake and carriage. Nanotoxicology. 2011;5:592-605.

111. Shah L, Yadav S, Amiji M. Nanotechnology for CNS delivery of bio-therapeutic agents. Drug Deliv Transl Res. 2013;3:336-351.

112. Vilella A, Tosi G, Grabrucker AM, et al. Insight on the fate of CNS-targeted nanoparticles. Part I: Rab5-dependent cell-specific uptake and distribution. J Control Release. 2013;174:195-201.

113. Hu YL, Gao JQ. Potential neurotoxicity of nanoparticles. Int J Pharm. 2010;394:115-121.
International Journal of Nanomedicine

\section{Publish your work in this journal}

The International Journal of Nanomedicine is an international, peerreviewed journal focusing on the application of nanotechnology in diagnostics, therapeutics, and drug delivery systems throughout the biomedical field. This journal is indexed on PubMed Central,

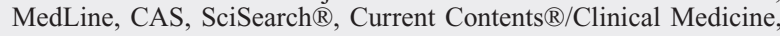

\section{Dovepress}

Journal Citation Reports/Science Edition, EMBase, Scopus and the Elsevier Bibliographic databases. The manuscript management system is completely online and includes a very quick and fair peer-review system, which is all easy to use. Visit http://www.dovepress.com/ testimonials.php to read real quotes from published authors.

Submit your manuscript here: http://www.dovepress.com/international-journal-of-nanomedicine-journal 